\title{
Molecular modeling with the aim: innovative approaches for drug design
}

\section{Dusanka Janezic}

Faculty of Mathematics, Natural Sciences and Information Technologies, University of Primorska, Koper, Slovenia.

We have developed new computational tools at the molecular scale for protein-ligand binding in drug discovery, ProBiS Tools (algorithm, database, and web servers) for predicting and modeling of medically interesting proteins based on graph theoretical approaches, combined with molecular simulations based on our earlier developed fast maximum clique algorithm, for finding a maximum clique in an undirected graph that is up to 100 times faster than the best comparable algorithm. ProBiS Tools opens a whole new range of possibilities in drug discovery.

Our newly developed approach is particularly useful in the context of precision medicine. Precision medicine promises more effective and safer treatments with lesser side effects. It depends on the advances within genomics, proteomics and transcriptomics fields that generate extensive amounts of data as well as it depend on the advances of computational biology. Our aim is extensive integration of the genomic, proteomic, and system level information (Figure 1) that enable evaluation of drug's effects on each and every person individually based on his/her genetic make-up.

Our tools enable joining several otherwise disconnected areas of research, i.e., genome sequence studies, protein structures, and MD simulations.

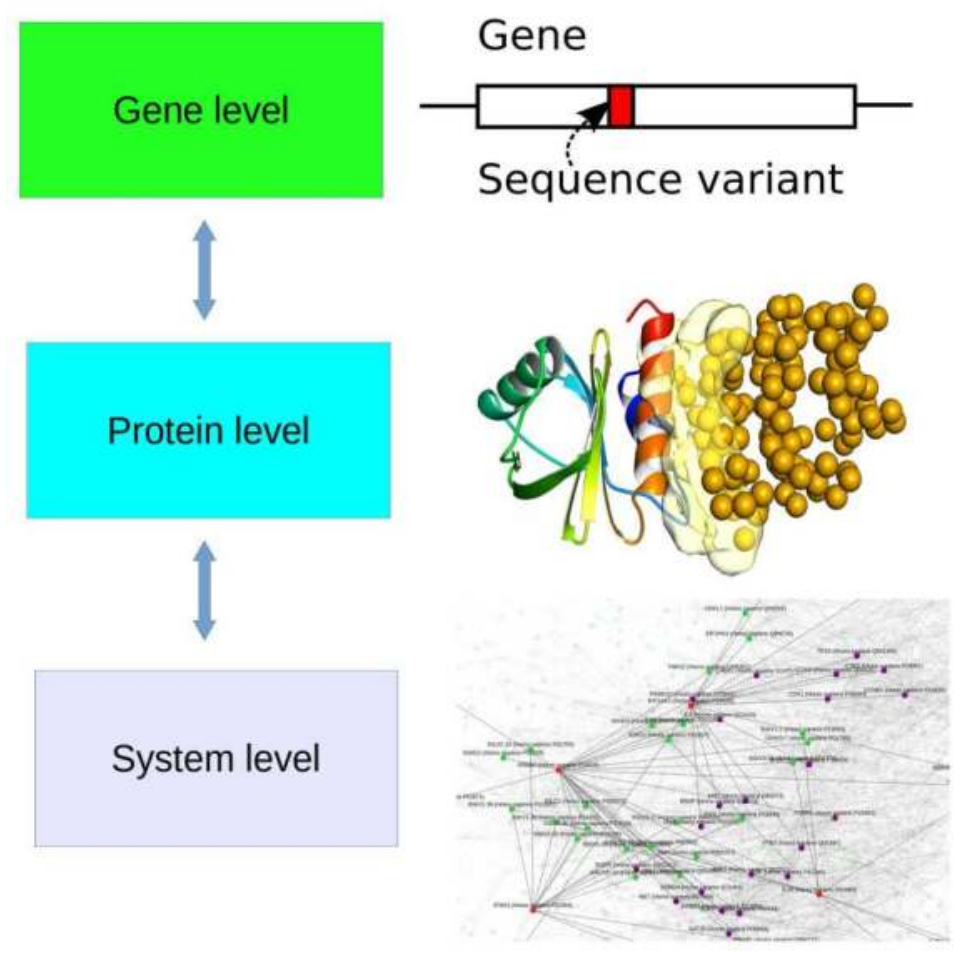

Description and tools:

1. Mapping of sequence variants to protein binding sites

(GenProBiS)

2. Prediction of binary proten-ligand interactions

(ProBiS, ProBis-ligands, ProBiS H2O)

3. Molecular simulations to study binding site dynamics (ProBiS-CHARMMing, CHARMM)

4. Protein interaction atlas for prediction of genetic variations involved in drug interactions and disease development (ProBiS-ATLAS)

Figure 1: Innovative approaches for drug design. 